\title{
The Effect of Raised Preoperative Serum Glucose Levels on Outcomes of Cataract Surgery
}

\author{
Sudhir Pendke, Iqbal Bombaywala, Saloni Singh \\ Department of Ophthalmology, Shri Vasantrao Naik Government Medical College Yavatmal, Maharashtra University of Health Sciences, \\ Maharashtra, India
}

Email address:

sudhirpendke@rediffmail.com (S. Pendke), Iqbal20069@rediffmail.com (I. Bombaywala)

\section{To cite this article:}

Sudhir Pendke, Iqbal Bombaywala, Saloni Singh. The Effect of Raised Preoperative Serum Glucose Levels on Outcomes of Cataract Surgery. International Journal of Ophthalmology \& Visual Science. Vol. 4, No. 3, 2019, pp. 58-62. doi: 10.11648/j.ijovs.20190403.14

Received: July 16, 2019; Accepted: August 21, 2019; Published: September 5, 2019

\begin{abstract}
Background: Cataract in diabetic patients is a major cause of visual impairment in the world. The aim of cataract surgery in diabetics with raised serum glucose levels is to achieve good postoperative vision and reduced complications. Purpose: The purpose of this study is to observe the intraoperative and postoperative complications and postoperative visual acuity after cataract surgery with intraocular lens implantation in diabetic patients with raised preoperative random blood sugar levels. Materials and method: A single surgeon carried out manual small incision cataract surgery followed by implantation of a posterior chamber intraocular lens. Patients were followed up at day 1, 6 weeks, 6 months and 1 year after surgery. At every visit Best Corrected Visual Acuity, measurement of intraocular pressure, anterior segment examination by slit lamp and dilated fundus examination was done. Result: Maximum number of patients was having excellent visual acuity (85.3\%) at 6 weeks follow up and in accordance to WHO recommendations. The most common postoperative complications were early postoperative fibrinous exudates $(28.2 \%)$, cystoid macular edema $(23 \%)$, posterior capsular opacification (20.5\%). Conclusion: In this study, we observed that over one year period, diabetic patients with raised serum glucose levels who underwent cataract surgery did not show any vision threatening complications. There was no influence of preoperative serum glucose levels on final visual outcome.
\end{abstract}

Keywords: Cataract, Diabetes Mellitus, Complications, Visual Outcome

\section{Introduction}

Diabetes Mellitus (DM) refers to a group of common metabolic disorders that share the phenotype of hyperglycemia. Criteria for the Diagnosis of DM [1]:Symptoms of diabetes plus random blood glucose concentration $\geq 11.1 \mathrm{mmol} / \mathrm{L} \quad(200 \mathrm{mg} / \mathrm{dl})$ or Fasting plasmaglucose $\geq 7.0 \mathrm{mmol} / \mathrm{L} \quad(126 \mathrm{mg} / \mathrm{dl}) \quad$ orHbAl $\mathrm{c} \geq 6.5 \%$ or Two hour plasma glucose $\geq 11.1 \mathrm{mmol} / \mathrm{L}(200 \mathrm{mg} / \mathrm{dl})$ during an oral glucose tolerance test.

Three important micro vascular complications of DM are neuropathy, nephropathy and diabetic eye disease. Among various eye manifestations, cataract and diabetic retinopathy constitutes major portion of avoidable blindness. Worldwide, more than 285 million people are affected by diabetes mellitus. In India, approximately 69 million people are affected by diabetes mellitus in 2015 [2]. Individuals with $\mathrm{DM}$ are 25 times more likely to become legally blind than individuals without DM [3]. Cataract is one of the major causes of visual impairment in diabetic patients [4]. In our rural population, most of the patients are unaware of this chronic disease and fail to receive timely treatment.

Diabetes affects all parts of the eye. Corneal manifestations include recurrent erosions, delayed wound healing, ulcers, and reduced corneal sensations. Diabetes also predisposes to cataract, glaucoma, rubeosis iridis, diabetic retinopathy, diabetic macular edema. Diabetics are more prone to develop cataract than others and at an early age also. Various mechanisms have been proposed for the pathogenesis of cataract in diabetes mellitus. In the lens, sorbitol is produced faster than it is converted to fructose by enzyme sorbitol dehydrogenase, a process that is increased in diabetics compared to nondiabetics [5]. In diabetics, lens opacities often progress following vitrectomy [6]. Snowflake cataract is seen commonly in type 1 diabetics. However, the most frequent type of cataract seen in diabetics is senile type 
[7]. Posterior subcapsular cataract has been shown to be significantly common in diabetics [8]. Cataract surgery in diabetics is indicated for visual improvement or to allow assessment and treatment of retinopathy [9]. The recent introduction of optical coherence tomography (OCT), especially spectral domain models, into clinical practice has improved the noninvasive monitoring of retinal thickness changes in diabetic retinopathy including postcataract surgery [10].

Also, some intraoperative complications are more common in diabetics. Anterior capsular phimosis is common in diabetics [11]. Diabetes patients are more prone to develop posterior capsular opacification than non-diabetic [12]. There is also evidence of poor pupillary dilatation in diabetics [13].

Intraoperative hyphema risk is also more due to presence of rubeosisiridis [14]. In diabetics corneal wound healing is delayed and corneal endothelial loss is more common in diabetics than non diabetics [15-18]. The impaired corneal wound healing has multiple etiologies including neurogenic (subbasal nerve abnormalities) and impaired corneal stem cell and epithelial cell division [19].

Patients with diabetes also have impaired immunity so more prone to postoperative infections.

\section{Materials and Methods}

This study was conducted at Shri Vasantrao Naik Government Medical College, Yavatmal, Maharashtra, India between December 2017 to December 2018. Total of 200 patients oftype 2 diabetes mellitus were included in the study. The patients were either previously diagnosed diabetics or newly detected by blood sugar levels. The cataract grading was done with Lens Opacification Classification System 3 (LOCS 3).

Informed consent was taken, patient's history and examination was done. Standard ocular examination included Best Corrected Visual Acuity (BCVA), slit lamp biomicroscopy, measurement of intraocular pressure and fundus examination. OCT was done if indicated to rule out any macular pathology. Patients were posted for manual small incision cataract surgery with clear corneal incision (incision length was $5.5-6.5 \mathrm{~mm}$ ), followed by a posterior chamber intraocular lens implantation. Patients were followed up at day 1, 6 weeks, 6 months and 1 year after surgery. At every visit Best Corrected Visual Acuity, measurement of intraocular pressure, anterior segment examination by slit lamp and dilated fundus examination was done.

Inclusion criteria: All patients of Type2 DM with senile cataract coming to our hospital who underwent manual small incision cataract surgery.

Exclusion criteria: All patients with diabetic retinopathy, diabetic maculopathy, glaucoma, uveitis, traumatic cataract, hypertensive retinopathy, history of previous ocular surgery of study eye were excluded.

Ethical clearance: After approval from ethical committee.

\section{Results}

In this study, Out of 200 diabetic patients, maximum (124) patients were having random blood sugar levels between 200-300mg/dl. The upper limit for random blood sugar level in this study is $350 \mathrm{mg} / \mathrm{dl}$.

Table 1. Preoperative random blood sugar levels.

\begin{tabular}{lll}
\hline Random blood sugar level (mg/dl) & Number of cases & Percentage (\%) \\
\hline $110-200$ & 57 & 28.5 \\
$200-300$ & 124 & 62 \\
$300-350$ & 19 & 9.5 \\
Total & 200 & 100 \\
\hline
\end{tabular}

Table 2. Age and sex distribution.

\begin{tabular}{lll}
\hline \multirow{2}{*}{ Age (years) } & Sex Distribution & \\
\cline { 2 - 3 } & Male & Female \\
\hline $50-60$ & 22 & 13 \\
$61-70$ & 73 & 44 \\
$71-80$ & 28 & 07 \\
$>81$ & 11 & 02 \\
TOTAL $=200$ & $134(67 \%)$ & $66(33 \%)$ \\
\hline
\end{tabular}

Table 3. Preoperative visual acuity.

\begin{tabular}{lll}
\hline Visual Acuity & Number of Cases & Percentage (\%) \\
\hline Light perception-hand movement & 16 & 08 \\
$1 / 60-5 / 60$ & 178 & 89 \\
$\geq 6 / 60$ & 06 & 03 \\
TOTAL & 200 & 100 \\
\hline
\end{tabular}

Preoperative visual acuity in $89 \%$ patients was in range of $1 / 60-5 / 60$. $8 \%$ patients were having mature cataract with visual acuity oh light perception to hand movement.

Table 4. Postoperative visual acuity.

\begin{tabular}{|c|c|c|c|c|c|}
\hline \multirow{2}{*}{ Who category } & \multicolumn{3}{|l|}{ Day one } & \multicolumn{2}{|l|}{ Six weeks } \\
\hline & Snellen acuity & n (\%) & Who recommendation (\%) & n (\%) & Who recommendation $(\%)$ \\
\hline 1 & $6 / 6-6 / 18$ & $119(59.5)$ & 40 & $157(85.3)$ & 85 \\
\hline 2 & $6 / 24-6 / 60$ & $59(29.5)$ & 50 & $22(11.9)$ & 10 \\
\hline $3+4$ & $<6 / 60$ & $22(11)$ & 10 & $5(2.7)$ & 5 \\
\hline TOTAL & $200(100)$ & & & $184(100)$ & \\
\hline
\end{tabular}

Maximum number of patients was having excellent visual acuity $(85.3 \%)$ at 6 weeks follow up and in accordance to WHO recommendations.

This finding is supported by previous reports, that those with maculopathy and retinopathy may have a valuable visual improvement after cataract surgery [20]. Further in a study, they have said that extracapsular cataract extraction with intraocular lens implantation is well tolerated in diabetics [21] with an overall good visual outcome [22] as evidenced by an $84.2 \%$ rate of improvement in preoperative 
visual acuity which correlates well with this study also. In this study we found that progression of diabetic retinopathy was not correlated with cataract surgery but with the natural course of diabetic vascular disease. A study done by Ostri et al they concluded that the cataract surgery improved the final visual outcome in patients with Diabetic Retinoapthy and cataract regardless of the degree of Diabetic Retinopathy and the apparent progression of Diabetic Retinopathy reflect the masking of low grades of Diabetic Retinopathy by preoperative lens opacities [23].

Table 5. Complications.

\begin{tabular}{lll}
\hline Complications & Number of Patients & Percentage (\%) \\
\hline Posterior capsular rent & 7 & 17.9 \\
Vitreous loss & 4 & 10.2 \\
Fibrinous exudates & 11 & 28.2 \\
$\begin{array}{l}\text { Posterior capsule } \\
\text { opacification }\end{array}$ & 8 & 20.5 \\
$\begin{array}{l}\text { Postoperative cystoid macular } \\
\text { edema }\end{array}$ & 9 & \\
Non healing wound & 0 & 23 \\
Endophthalmitis & 0 & 0 \\
& 39 & 0 \\
\hline
\end{tabular}

The most common intraoperative complication in this study was posterior capsular rent followed by vitreous loss. A similar result was obtained by a study conducted by Oluwatoyin $\mathrm{H}$ et al in diabetic patients [24].

The most common postoperative complications were early postoperative anterior segment inflammation, Posterior capsular opacification and cystoid macular edema. Similar results were seen by Menchini et al. they reported intraocular inflammation and its sequelae as the most common complication in their study [25]. Ivancic et al. reported that inflammatory reactions and bleeding which resulted in postoperative keratopathy, fibrinous uveitis and posterior capsule opacity as the common complications of cataract surgery amongst diabetics [26]. Till now, no studies have concluded a definite role of perioperative glycemia on cystoid macular edema rate [27]. These inflammatory complications were resolved in further follow-ups of patients and overall visual prognosis was good at the end of one year irrespective of poor glycemic control.

\section{Discussion}

In developing countries like India patients often present late for medical care with subsequent delay in diagnosis and treatment. In this study, the indication of cataract surgery was achievement of good postoperative vision. Diabetic patients presenting with dense cataract were benefited by cataract surgery followed by post-operative assessment and treatment of retinopathy. In this study we did not see any patient of postoperative endophthalmitis or poor wound healing in diabetics. Since, the role of perioperative hyperglycemia remains controversial in causation of postoperative endophthalmitis in patients of diabetes mellitus [28, 29], we cannot conclude that higher levels of blood glucose poses a higher incidence of endophthalmitis or poor wound healing in diabetics.

A study involving 50 patients was studied by Krepler in which no influence on the progression of diabetic retinopathy and visual improvement was achieved in majority of patients with Non Proliferative Diabetic Retinopathy but poorer visual outcome was observed in patients developing macular edema following cataract surgery [30]. Rapid pre-operative gylcemic control should be avoided as it may increase the risk of postoperative progression of retinopathy and maculopathy [31]. Cataract surgery can be performed in patients of moderate to severe non proliferative Diabetic Retinopathyinstead of poor glycemic control.

The reason for poor postoperative visual acuity in our study was posterior capsule opacification and postoperative cystoid macular edema. Nascimento et al. reported that serum glucose level had no influence on peri-operative clinical complications and final visual outcome of cataract surgery amongst diabetic patients [23].

\section{Conclusion}

There are many considerations that must be addressed when performing cataract surgery in patients with diabetes with raised serum glucose levels. Major issues are aqueous humour inflammation, Posterior capsular opacification, progression of Diabetic Retinopathy, and development or worsening of macular edema. Presence of Diabetic Retinopathy is not a contraindication for cataract surgery but those with Diabetic Retinopathy should be closely monitored in postoperative period for early signs of progression. Cataract surgery in diabetics has an overall good visual outcome and should not be denied surgery irrespective of poor glycemic control. Modern surgical and pharmacologic therapies allow safer and effective surgery in diabetics even in raised serum glucose levels. Inspite of poor glycemic control there were no serious postoperative complications in our study. This study did not show any influence on final visual acuity in diabetes patients. Many studies have also shown that the progression of Diabetic Retinopathy is related to the natural evolution of the disease rather than the role of cataract surgery itself. The postoperative final visual acuity and complications showed no correlation with the glycemic control in our study.

As cataract surgery is done under local anesthesia, stringent criteria for perioperative blood sugar should not be considered for postponement of the surgery. Up to date, the clinical evidences are lacking regarding tight intraoperative glucose control in mitigating the intraoperative and postoperative complications of cataract surgery.

\section{Recommendations}

By this study the author recommends that patients with raised preoperative serum blood glucose levels should not be denied of cataract surgery as there was no influence of uncontrolled sugar levels on complications and visual 
outcome after cataract surgery. Still, further studies and more number of patients needed to be observed in this aspect.

\section{Conflict of Interest}

All the authors do not have any conflicts of interest.

\section{References}

[1] Harrison's Principles of Internal Medicine $18^{\text {th }}$ edition.

[2] International Diabetes Federation (IDF). Diabetes Atlas $7^{\text {th }}$ Edition, International Diabetes Federation Brussel, Belgium. 2015. http://www.diabetesatlas.org.

[3] Ebihara Y, Kato S, Oshika T, Yoshizaki M, Sugita G. posterior capsule opacification after cataract surgery in patients with diabetes mellitus cataract refract surg 2006: 32 (7): 1184-7.

[4] Drinkwater JJ, Davis WA, Davis TME. A systemic review of risk factors for cataract in type 2 diabetes. Diabetes Metab Res Rev. 2019; 35: e3073.

[5] Kador PF, Wyman M, Oates PJ. Aldose reductase, ocular diabetic complications and development of topical Kinostat. ProgRetin Eye Res 2016; 54: 1-29.

[6] Kataria AS, Thompson JT. Cataract formation and progression in patients less than 50 years of age after vitrectomy. Ophthalmol Retina 2017; 1: 149-53.

[7] Pollreisz A, Schmidt-Erfurth U. Diabetic cataractpathogenesis, epidemiology and treatment. J Ophthalmol 2010; 2010: 608751 .

[8] Rowe NG, Mitchell PG, Cumming RG, Wans JJ. Diabetes, fasting blood glucose and age-related cataract: The Blue Mountains Eye Study. Ophthalmic Epidemiol 2000; 7: 103-14.

[9] Cunliffe IA, Flanagan DW, George NDL, Aggarwaal RJ, Moore AT. Extracapsular cataract surgery with lens implantation in diabetics with or without proliferative retinopathy. Br J Ophthalmol. 1991; 75: 9-12.

[10] Denniston AK, Chakravarthy U, Zhu H, Lee AY, Crabb DP, Tufail A, et al. The UK diabetic retinopathy electronic medical record (UK DR EMR) users group, report 2: Real-world data for the impact of cataract surgery on diabetic macular edema. Br J Ophthalmol 2017; 101: 1673-8.

[11] Zaczek A, Zetterstorm C. posterior capsule opacification after phacoemulsification in patients with diabetes mellitus cataract Refract surg 1999: 25 (2): 233-7.

[12] Ebihara Y, Kato S, Oshika T, Yoshizaki M, Sugita G. Posterior capsule opacification after cataract surgery in patients with diabetes mellitus. J Cataract Refract Surg 2006; 32: $1184-7$.

[13] Cahill M, Eustace P, de Jesus V. Pupillary autonomic denervation with increasing duration of diabetes mellitus. Br J Ophthalmol. 2001; 85: 1225-30.

[14] Jardeleza MS, Miller JW. Review of anti-VEGF therapy in proliferative diabetic retinopathy. SeminOphthalmol 2009; 24: 87-92.
[15] Shih KC, Lam KS, Tong L. Asystematic review on the impact of diabetes mellitus on the ocular surface. Nutr Diabetes 2017; 7: e251.

[16] Morikubo S, Takamura Y, Kubo E, Tsuzuki S, Akagi Y. Corneal changes after small-incision cataract surgery in patients with diabetes mellitus. Arch Ophthalmol 2004; 122: 966-9.

[17] Hugod M, Storr-Paulen A, Norregaard JC, Nicolini J, Larsen $\mathrm{AB}$, Thulsen $\mathrm{J}$, et al. Corneal endothelial cell changes associated with cataract surgery in patients with type 2 diabetes mellitus. Cornea 2011; 30: 749-53.

[18] Yang R, Sha X, Zeng M, Tan Y, Zheng Y, Fan F, et al. The influence of phacoemulsification on corneal endothelial cells at varying blood glucose levels. Eye Sci 2011; 26: 91-5.

[19] Shih KC, Lam KS, Tong L. A systemic review on the impact of diabetes mellitus on the ocular surface. Nutr Diabetes 2017; 7: e251.

[20] Cunliffe IA, Flanagan DW, George NDL, Aggarawaal RJ, Moore AT. Extracapsular cataract surgery with lens implantation in diabetics with or without proliferative retinopathy. Br J Ophthalmol. 1991; 75: 9-12.

[21] Gabric N, Henc-Petrinovic L, Dekaris I, Busic M, PtrinovicDoresic J. Timing of cataract surgery in diabetics. Acta Med Croatia. 1996; 50: 25-8.

[22] Mechini U, Cappelli S, Virgili G. Cataract surgery and diabetic retinopathy. SeminOphthalmol. 2003; 18: 103-8.

[23] Ostri C, Lund Andersen H, Sander B, La Cour M. Phacoemulsification cataract surgery in a large cohort of diabetes patients; visual acuity outcomes and prognostic factors. J Catarct Refract Surg. 2011; 37 (11); 2006-12.

[24] Oluwatoyin H, Onakpoya, Charles 0, Bekibele, and Stella A. Adegbehingbe. Cataract Surgical Outcomes In Diabetic Patients: Case Control Study. Middle East Afr J Ophthalmol. 2009 Apr-Jun; 16 (2): 88-91.

[25] Mechini U, Cappelli S, Virgili G. Cataract surgery and diabetic retinopathy. SeminOphthalmol. 2003; 18: 103-8.

[26] Ivancic D, Mandic Z, Brac C, Kopic M. Cataract surgery and post operative complication in diabetic patients. CollAntropol. 205; 29 : 55-8.

[27] Wielders LHLambermontVASchouten JS, et al. Prevention of cystoids macular edema after cataract surgery in nondiabetic and diabetic patients: a systemic review and meta-analysis. Am J Ophthalmol 2015; 160: 968-81.

[28] El-MollayessGMSaadehJSSalti HI. Exogenous endophthalmitis in diabetic patients: a systemic review. ISRN Ophthalmol 2012; $456209 . \quad$ doi: 10.5402/2012/456209.eCollection 2012.

[29] JabbarvandMHashemianHKhodaparast M JouhariMTabatabaeiARezaei S. Endophthalmitis occurring after cataract surgery: outcomes of more than 480000 cataract surgeries, epidemiologic features, and risk factors. Ophthalmology 2016; 123: 295-301.

[30] Krepler K, Biowski R, Schrey S, Jandrasits K, Wedrich A. Cataract surgery in patients with diabetic retinopathy: visual outcome, progression ofdiabetic retinopathy, and incidence of diabetic macular edema. Grafes Arch ClinExpOphthalmol. 2002; 240 (9); 735-738. 
[31] Hjortrup A, Sorensen C, Dyremose E, Hjortso NC, Kehlet H. Influence of diabetes mellitus on operative risk. Br J Surg. 1985; 72 (10); 783-5.
[32] Nascimento MA, Lira RP, Kara-Jose n, Arieta CE. Predictive value of preoperative fasting glucose test of diabetic patients regarding surgical outcome in cataract surgery. Arq Bras Oftalmol. 2005; 68: 213-7. 